\title{
1 Serum extracellular vesicles trace COVID-19 progression and immune 2 responses
}

5 Kevin Ho Wai Yim ${ }^{1}$, Simone Borgoni ${ }^{1}$ and Richard Chahwan ${ }^{1 *}$

6

7

$8{ }^{1}$ Institute of Experimental Immunology, University of Zurich, Zurich, Switzerland

13 * Corresponding author: Richard Chahwan (chahwan@immunology.uzh.ch)

16 Keywords: COVID-19, SARS-CoV-2, extracellular vesicles, diagnosis, clinical liquid biopsy

19 Word count: 5052

20

21 


\section{ABSTRACT}

23 Coronavirus disease 2019 (COVID-19) has transformed very quickly into a world pandemic with

24 severe and unexpected consequences on human health. Concerted efforts to generate better

25 diagnostic and prognostic tools have been ongoing. Research, thus far, has primarily focused

26 on the virus itself or the direct immune response to it. Here, we propose extracellular vesicles

27 (EVs) from serum liquid biopsies as a new and unique modality to unify diagnostic and 28 prognostic tools for COVID-19 analyses. EVs are a novel player in intercellular signaling 29 particularly influencing immune responses. We herein show that innate and adaptive immune 30 EVs profiling, together with SARS-CoV-2 Spike $\mathrm{S} 1^{+}$EVs provide a novel signature for COVID-

3119 infection. It also provides a unique ability to trace the co-existence of viral and host cell 32 signatures to monitor affected tissues and severity of the disease progression. And provide a 33 phenotypic insight into COVID-associated EVs. 


\section{INTRODUCTION}

38 Coronavirus disease 2019 (COVID-19) was first identified in December 2019 in Wuhan, China.

39 The disease progressed into a global pandemic with over 64 million confirmed cases and over

403.9 million confirmed deaths as of June $2021^{[1]}$. COVID-19 patients can be asymptomatic, suffer

41 from mild symptoms such as fever, cough, and dyspnea or develop into severe conditions

42 characterized as acute respiratory distress syndrome (ARDS) requiring mechanical ventilation ${ }^{[2]}$.

43 SARS-CoV-2, a positive-sense, single-stranded RNA virus, is known as the causative pathogen

44 of COVID-19. The commonly acknowledged mechanism of SARS-CoV-2 pathology is the entry

45 of viruses into angiotensin-converting enzyme 2 (ACE2) expressing host cells, with a tropism for

46 different organs, such as the respiratory tract, kidneys, liver, heart, brain, and blood vessels ${ }^{[3]}$.

47 SARS-CoV-2 infected cells can recruit different immune cell types and induce innate

48 inflammatory responses as well as adaptive immune responses mediated by targeted

49 antibodies. Sars-CoV-2 specific immunoglobulins ( $\mathrm{lg}$ ) types $\mathrm{M}, \mathrm{A}$, and $\mathrm{G}$ have been used as an

50 indicator of protective immunity in infected patients. However, such antibody responses

51 normally emerge around 10 to 21 days after infection and may take even longer (four weeks or

52 more) in mild cases to be detected ${ }^{[4]}$. In general, around $5 \%$ of COVID-19 patients develop

53 severe conditions like ARDS, which arises around one week after symptom onset. Therefore,

54 SARS-CoV-2 specific antibody titer measurement is not the best predictor of severe disease for

55 infected patients who show mild symptoms early after infection but rapidly develop ARDS.

56 Extracellular vesicles (EVs) are nanosized lipid-bilayer vesicles that carry nucleic acid and

57 protein cargo. They are constitutively secreted by virtually all types of cells and circulate in most

58 biofluids such as blood, urine, saliva, and breast milk. Since the surface markers and molecular

59 cargo of EVs can reflect the cellular origin and activation status, they have been utilized as non-

60 invasive biomarkers from liquid biopsies in the past decade, for diagnostic and prognostic

61 purposes ${ }^{[5-7]}$. Thanks to the lipid-bilayer structure of EVs, they are intrinsically more stable than

62 naked circulating molecules such as antibodies and cytokines, conferring a higher potential to 
63 provide a more robust and long-lasting effect on the host immune response. Here, we

64 characterized serum EVs from healthy donors, early COVID-19 patients $(<13$ days from

65 symptom onset), and late COVID-19 patients with mild disease (> 13 days from symptom onset),

66 in terms of size distribution, concentration and surface marker profile using nanoparticle flow

67 analyzer $\left(\mathrm{NanoFCM}^{\circledR}\right)^{[8]}$. Cluster analysis of different EVs subpopulations based on surface

68 marker expression was performed to identify signatures of healthy donors, early COVID-19 and

69 late COVID-19 patients. Lastly, COVID-19 specific SARS-CoV-2 Spike S1 ${ }^{+}$serum EVs were

70 characterized in relation to disease progression and host immune responses to determine

71 disease severity.

\section{RESULTS}

75 Multiplex profiling of serum EVs derived from mild COVID-19 patients

76 To explore the landscape of serum immune EVs during SARS-CoV-2 infection, we sampled a

77 cohort of 20 mild COVID-19 patients and 17 healthy donors (Table 1). According to the WHO

78 definition (World Health Organization, 2020), all the sampled COVID-19 patients in this study

79 experienced mild illness with symptoms such as fever, fatigue, or dyspnea. Serum EVs were

80 isolated from the whole blood of donors and patients. Purified serum EVs were analyzed by

81 nanoparticle analyzer to examine the size distribution and concentration of different serum EVs

82 subsets with a dedicated panel of immune markers and tetraspanins marker (Fig. 1A).

83 According to MISEV 2018 guideline, the physical properties of EVs, such as particle size

84 distribution and concentration were analyzed to ensure the reproducibility of the result.

85 Moreover, for diagnostic interest, potential variance in particle size distribution of different serum

86 EVs subsets between healthy controls and COVID-19 patients might provide valuable predictive

87 information (Fig. S1a). Using a mixture of four different sizes $(66,91,113,155 \mathrm{~nm})$ of

88 monodisperse silica nanoparticles (refractive index $=1.461$ ), we applied 4 size interval bins 
based on the 4 separated side-scatter burst areas to quantify the approximate size distribution

90 of serum EVs, abbreviated as small, medium, large, extra-large accordingly (Fig. S1b). In

91 COVID-19 patients small EVs were more enriched, and CD66 $\mathrm{b}^{+}$EVs showed an increase

92 compared to healthy controls ( $p<0.05, p<0.01$ respectively). However, extra-large EVs were

93 reduced in $\mathrm{CD} 3^{+}, \mathrm{CD} 8^{+}, \lg \mathrm{A}^{+}, \lg \mathrm{G}^{+} \mathrm{EVs}$ in COVID-19 patients compared to healthy controls $(\mathrm{p}$

$94<0.05)$ (Fig. 1B, C, S1d).

95 Immune cells profiling of COVID-19 patients revealed numerous alterations in both innate and

96 adaptive immunity. However, whether immune cells derived EVs were influenced by COVID-19

97 or involved in any form of disease specific responses remains unknown. Canonical leukocyte

98 marker CD45 was first examined in patient serum EVs to gain an overview of immune EVs

99 changes in COVID-19. Interestingly, CD $45^{+}$serum EVs showed a significant reduction in mild

100 COVID-19 patients compared to healthy control (Fig. S1c). We next visualized the alteration of

$101 \mathrm{CD}^{2} 5^{+}$EVs levels against days post symptoms onset. Significant depletion in CD45 EVs in

102 patients between 3 to 13 days post symptoms onset was observed compared to healthy

103 controls $(p<0.05)$. Strikingly, patients after 13 days post symptoms onset displayed a recovery

104 of $\mathrm{CD} 45^{+} \mathrm{EVs}$ level comparable to healthy controls. This finding highlighted the importance to

105 dissect the analysis into pre- and post-13 days post symptoms onset to gain more precise

106 perspectives in the serum EVs dynamics in COVID-19. Interestingly, depletion of CD45 EVs in

107 the early onset of COVID-19 correlated to $\mathrm{CD} 45^{+}$cells deficiency observed in severe COVID-19

108 patients reported by another study ${ }^{[9]}$, indicating the high sensitivity and early detection capacity

109 of EVs based diagnostics. Total EVs concentration did not show significant differences

110 suggesting the alteration in $\mathrm{CD} 45^{+} \mathrm{EVs}$ level was independent of total EVs production during

111 SARS-CoV-2 infection (Fig. 1C, D). The correlation between sizes and markers in EVs subsets

112 between healthy controls and COVID-19 patients was visualized by Spearman's rank correlation

113 matrix (Fig. 1E). A significant correlation of large and extra-large CD31 ${ }^{+}$EVs (predominantly

114 expressed by endothelial tissues) and total EVs was observed in healthy controls and post-13 
115 days patients but not in pre-13 days patients. There was also a strong correlation between the 116 small CD14 ${ }^{+}, \mathrm{CD}_{19}{ }^{+}, \mathrm{CD}^{+} 6^{+}$and $\mathrm{CD}^{+} 3^{+} \mathrm{EVs}$ in pre-13 days patients which were not present in

117 healthy controls nor post-13 days patients. These data suggest that classical monocytes-, B 118 cells- and natural killer cells-derived small EVs are predominantly affected in the early stage of 119 COVID-19.

121 Detection of SARS-CoV-2-Spike S1+ serum EVs

122 ACE2 containing EVs have been reported to prevent infection by SARS-CoV-2 virus indicating 123 the relevance of EVs in COVID-19 progression ${ }^{[10]}$. Moreover, SARS-CoV-2 Spike S1 containing 124 EVs have been shown to serve as decoys for neutralizing antibodies ${ }^{[11]}$. To explore whether 125 SARS-CoV-2 utilizes such strategies to influence disease progression and interaction with the 126 host, we attempted to detect and profile Spike $\mathrm{S}^{+}$serum EVs in this cohort of patients. To 127 eliminate the possibility of virus contamination in serum EVs purification, viral RNA detection by 128 PCR was performed with patients' serum and none of the specimens tested positive. Specificity 129 of Spike S1 antibody has been validated with negative control HEK293A and Spike S1 130 transfected HEK293A and their released EVs, in combination with serial titration of staining 131 cocktail with recombinant Spike S1 proteins (Fig. S2).

132 Spike $\mathrm{S} 1^{+} \mathrm{EVs}$ were detected in a small subset of healthy controls, suggesting their serum EVs 133 might be carrying cross-reactive epitopes that bind to Spike S1 antibodies used in the study. We, 134 therefore, applied a cut-off of Spike S1 fluorescence signal at $1.75 \%$ of total particles to exclude 135 all healthy controls. 5 out of 16 mild COVID-19 patients (dark blue dots in plots) had $>1.75 \%$ 136 Spike $\mathrm{S}^{+}$serum EVs (classified as Spike $\mathrm{S} 1^{+} \mathrm{EVs}$ positive), suggesting the existence of SARS137 CoV-2 specific EVs in human serum which could subsequently influence disease progression 138 (Fig. 2A). To further identify the origin of Spike S1 ${ }^{+} \mathrm{EVs}$, we co-stained Spike S1 with a panel of 139 immune and endothelial markers since SARS-CoV-2 are known to significantly affect 140 endothelial tissues in patients ${ }^{[12]}$. Notably, Spike $S 1^{+} \mathrm{CD} 31^{+} \mathrm{EVs}$ levels were significantly 
141 increased in pre-13 days COVID-19 group compared to healthy controls and post-13 days

142 COVID-19, but not with the immune markers analyzed (Fig. S3). Elevated levels of endothelial 143 markers including CD31 were reported in COVID-19 ${ }^{[13]}$, and temporary stress triggers CD31+ 144 microparticle release ${ }^{[14]}$. These data suggest Spike $S 1^{+}$EVs likely originate from SARS-CoV-2 145 infected endothelial tissues. Total CD31+ EVs level also showed an elevating tendency in 146 COVID-19 patients compared to healthy controls. Reduction of small Spike S1+ EVs was also 147 observed in post-13 days patients compared to pre-13 days patients (Fig. 2B).

148 To gain deeper insights into the relationship between serum EVs and disease status, we applied 149 principal component analysis of serum EVs subset level across the cohort (Fig. 2C). A biplot 150 revealed the contribution of different EVs subsets as arrows showing the significant association 151 of $\mathrm{CD}_{4} 5^{+} \mathrm{EVs}$ and healthy controls. A quartile was dominated by pre-13 days patients and 152 highly associated with Spike $S 1^{+}$EVs. A quartile with mostly post-13 days was defined by high 153 levels of $\mathrm{CD}_{3} 1^{+}$and $\mathrm{CD} 3^{+} \mathrm{EVs}$. In another quartile, a heterogeneous mix of predominantly 154 healthy controls was defined by high levels of $\mathrm{CD}^{+}, \mathrm{CD} 14^{+}, \mathrm{CD} 19^{+}$and $\mathrm{CD} 56^{+}$EVs. Individuals 155 PCA clustering allowed the stratification of disease status based on frequencies of different EVs 156 subsets across the cohort. Taken together, despite the anticipated diversity across individuals 157 and less pronounced phenotypes in mild COVID-19 compared to severe ones, these 158 exploratory analyses enable the usage of serum EVs to predict disease status on top of 159 canonical clinical approaches (Fig. 2D).

160 To further probe the alterations of different serum EVs subsets during COVID-19, we applied 161 hierarchical clustering in a correlation map of serum EVs subsets across the cohort (Fig. 2E). In 162 the healthy controls group, specialized immune cell derived EVs, CD4 ${ }^{+}, \mathrm{CD}_{14}{ }^{+}, \mathrm{CD}_{19^{+}}$and $163 \mathrm{CD}^{2} 6^{+} \mathrm{EVs}$ had a strong positive correlation with each other but negatively correlate with $\mathrm{CD}^{+} 5^{+}$ 164 EVs, suggesting, in healthy state, immune EVs are less likely to originate from lymphocytes nor 165 monocytes but other types of leukocytes (i.e., neutrophils, around 60\% of leukocytes in blood). 166 However, such negative correlation did not persist in COVID-19 patients, indicating the surge of 
167 lymphocytes and monocytes driven responses during SARS-CoV-2 infection. Significant 168 positive correlation was also observed between $\mathrm{CD} 38^{+}$and $\operatorname{lgG}{ }^{+} \mathrm{EVs}$ in healthy controls but not 169 in any COVID-19 group. In the pre-13 days mild COVID-19 group, CD $31^{+}$and $\mathrm{CD} 63^{+} \mathrm{EVs}$ 170 started to positively correlate with specialized immune EVs. Strong positive correlation of Spike $171 \mathrm{~S}^{+}$and Spike $\mathrm{S} 1^{+} \mathrm{CD} 31^{+} \mathrm{EVs}$ and negative correlation of $\mathrm{CD} 19^{+}$and $\mathrm{CD} 66 \mathrm{~b}^{+} \mathrm{EVs}$ were also 172 observed. In the post-13 days mild COVID-19 group, the strong positive correlation between 173 specialized immune EVs was reduced as well as the correlation of Spike S1+ and Spike $174 \mathrm{~S} 1+\mathrm{CD} 31+\mathrm{EV}$ s. In the overall mild COVID-19 group, regardless of time after symptoms onset, 175 specialized immune EVs positively correlate with CD $31^{+}$EVs and Spike S1 ${ }^{+}$EVs also positively 176 correlate with Spike S1+CD31+ EVs. In summary, the analysis of serum EVs cluster signatures 177 in relation to disease status and time after symptoms onset uncovered the dynamic patterns of serum EVs subset levels during COVID-19 progression.

Abundance of SARS-CoV-2-Spike S1 serum EVs indicate host immunological responses

181 To better understand the dynamics of Spike S1+ EVs during SARS-CoV-2 infection, we 182 visualized the alteration of Spike $\mathrm{S} 1^{+}$and Spike $\mathrm{S} 1^{+} \mathrm{CD} 31^{+} \mathrm{EVs}$ across the cohort against time 183 after symptoms onset (Fig. 3A). Spike $\mathrm{S}^{+} \mathrm{EV}$ s positive patients were present mostly in pre-13 184 days post symptoms onset, two of which from day 10 showed high Spike $\mathrm{S} 1^{+} \mathrm{CD} 31^{+}$EVs (Fig. 185 3A). To explore the immunological relevance of serum Spike $S 1^{+}$EVs during COVID-19 186 progression, we analyzed the SARS-CoV-2 specific immunoglobulins levels across the cohort 187 and in relation to time after symptoms onset (Fig. 3B). SARS-CoV-2 specific IgA and IgG levels 188 were below $0.6 \mathrm{G} / \mathrm{l}$ in all healthy controls and showed an increasing trend close to 10 days post 189 symptom onset (Fig. 3B, 3C). Two of the Spike S1 $1^{+}$EVs positive patients at day 10 showed 190 elevated levels of SARS-CoV-2 specific $\lg A$ and $\lg G$ (up to 24 and $3 \mathrm{G} / \mathrm{l}$ respectively) which are 191 predominantly observed in severe COVID-19 cases, suggesting the dynamics Spike S1+ EVs 192 levels in patients could provide sensitive detection of alternated immunological responses. 
193 Levels of immunosuppressive cytokine $\mathrm{IL}-10^{[15]}$, one of the hallmarks observed in severe

194 COVID-19 was compared across the cohort. IL-10 levels were comparable between healthy 195 controls and COVID-19 patients, indicating cytokine secretion was not significantly affected in 196 this cohort of mild COVID-19 patients (Fig. 3D).

197 To further understand the relationship between Spike S1+ EVs and other significantly altered 198 factors between healthy controls and COVID-19 patients, we performed direct correlation 199 analysis between the levels of Spike $S 1^{+} \mathrm{EVs}$ and Spike $\mathrm{S} 1^{+} \mathrm{CD} 31^{+} \mathrm{EVs}$, viral specific 200 immunoglobulins (IgG1 and IgG3) and immunosuppressive IL-10 (Fig. 3E). A strong positive 201 correlation was observed between Spike $S 1^{+} \mathrm{EVs}$ and Spike $S 1^{+} \mathrm{CD} 31^{+} \mathrm{EVs}(r=0.6, \mathrm{p}=0.017)$, 202 suggesting the Spike $\mathrm{S1}^{+} \mathrm{EVs}$ are likely to originate from endothelial tissues. We also found a 203 strong negative correlation between Spike $S 1^{+} \mathrm{EVs}$ and $\lg \mathrm{G} 1(r=-0.46, \mathrm{p}=0.036)$ and $\lg \mathrm{G} 3(\mathrm{r}$ $204=-0.75, p=0.0013)$. In contrast to SARS-CoV-2 specific $\lg A$ and $\lg G$, $\lg G 1$ and $\lg G 3$ are 205 predominantly induced by viral infection, with IgG3 appearing first during infection ${ }^{[16]}$. These 206 negative correlations indicate levels of Spike $\mathrm{S1}^{+} \mathrm{EVs}$ could estimate whether the host antibody 207 mediated responses are SARS-CoV-2 specific. Another negative correlation was observed 208 between Spike $\mathrm{S1}^{+} \mathrm{EVs}$ and immunosuppressive IL-10, suggesting patients with higher Spike $209 \mathrm{S1}^{+} \mathrm{EVs}$ are less prone to immunosuppression of the immune system and possibly a lower 210 chance of disease deterioration.

212 Mild COVID-19 patients derived serum EVs affect healthy PBMCs responses ex vivo

213 Immune cells derived soluble factors such as IFN-y, IL6, and TNF are known to cause 214 inflammation in severe COVID-19 cases and subsequently cause complications in the disease. 215 Since EVs have been shown to modulate immune responses in viral infection, we sought to 216 explore the possibility of EVs mediated immune regulation in COVID-19. B cells mediated 217 antibodies neutralization and T cells mediated cytokines production are the key drivers of host 218 immune defenses against COVID-19, understanding B and T cells responses in the presence of 
219 purified serum EVs from the cohort would enable us to better understand the immune regulatory 220 network in COVID-19. To understand such mechanism, we activated healthy PBMC CD19+ B 221 cells (with IL-4 and IL-21) and $\mathrm{CD}^{+} \mathrm{T}$ cells (anti-CD3/CD28 and PMA/lonomycin) ex vivo in the 222 presence of PBS control and purified serum EVs across the cohort. Cell viability, activation, 223 antibodies class switching (in B cells), and cytokine production (in T cells) were measured 4 224 days after activation by flow cytometry (Fig. 4A).

225 Viability of $\mathrm{CD} 19^{+} \mathrm{B}$ cells was consistent across the serum EVs in the cohort. Activation marker 226 CD86 expression was reduced in pre-13 days COVID-19 derived EVs compared to both healthy 227 controls and post-13 days COVID-19 derived EVs, suggesting serum EVs from pre-13 days 228 suppress B cells activation. IgM expression was similar across the serum EVs in the cohort 229 while IgA expression was significantly increased in post-13 days COVID-19 EVs, implying post23013 days COVID-19 EVs promote antibodies class switching.

231 Viability of $\mathrm{CD}^{+} \mathrm{T}$ cells was reduced in pre-13 days COVID-19 derived EVs compared to post23213 days COVID-19 derived EVs but not observed in $\mathrm{CD}^{+} \mathrm{T}$ cells. Activation marker CD69 233 expression in $\mathrm{CD}^{+}$and $\mathrm{CD} 8^{+} \mathrm{T}$ cells was similar across the serum EVs in the cohort. Significant 234 reduction of TNF production in $\mathrm{CD}^{+} \mathrm{T}$ cells was observed in pre-13 days COVID-19 EVs, with a 235 reducing tendency in post- 13 days COVID-19 EVs compared to healthy controls. However, TNF 236 production in $\mathrm{CD}^{+} \mathrm{T}$ cells was comparable across the serum EVs in the cohort, suggesting $237 \mathrm{CD}^{+} \mathrm{T}$ cells are more susceptible to serum EVs modulation. IFN-y production of $\mathrm{CD}^{+}$and $238 \mathrm{CD}^{+} \mathrm{T}$ cells was increased in pre-13 days COVID-19 EVs compared to both healthy controls 239 and post-13 days COVID-19 derived EVs. Taken together, serum EVs from COVID-19 patients 240 are capable to influence B and T cells responses in a targeted manner that possibly contribute 241 to the immune phenotypes observed in COVID-19 patients (Fig. 4B).

\section{DISCUSSION}


245 Throughout the COVID-19 pandemic, immune cell-profiling studies continue showing a wide 246 spectrum of immunological complications from mild to severe cases such as lymphopenia, 247 hyper inflammation and series of cytokine storms. Aside from studied soluble immune factors 248 like antibodies and cytokines, EVs are emerging as novel and potent intercellular signaling 249 mediators. EV cargos are under study as predictive tools for COVID-19 severity ${ }^{[17-19]}$. However, 250 such recent studies required mass spectrometry and RNA-seq of EVs cargo which is not easy 251 to convert into routine clinical diagnostics due to the tedious processing and high expertise 252 required. Or they studied covid related EVs ex vivo ${ }^{[11]}$. We focus here on i) serum immune253 derived EVs because immune cells are predominantly found in the bloodstream, as are their 254 released EVs; ii) viral-specific EVs to gain specificity and viral tracking ability; iii) on analyzing 255 early- and late- mild covid patients to study the effect of the virus with minimal symptom 256 complications; and iv) attributing phenotypic functions for COVID-19 associated EVs. Therefore, 257 our analysis has consequences that surpass the basic investigation of COVID-19 biomarkers 258 per se and instead tries to trace the dynamic response of the virus and our body's response to it.

260 To harness the full potential of immune EVs in COVID-19, we applied multi-parametric EVs 261 phenotyping to characterize total EVs and specific immune associated subsets. The high 262 sensitivity and resolution of $\mathrm{NanoFCM}^{\circledR}$ used in this study allowed the possibility of direct single 263 EVs phenotyping. We could simultaneously measure and correlate EV size, concentration and 264 marker subset quantification; surpassing the conventional antibody coated beads approach 265 currently used ${ }^{[20]}$. General enrichment of small EVs $(\sim 66 \mathrm{~nm})$ in mild COVID-19 patients was

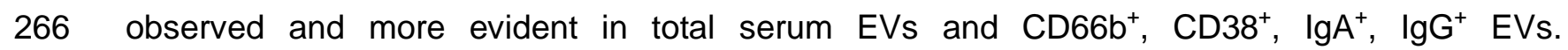
267 Interestingly, such enrichment of small EVs seems to correlate with reduction in extra-large EVs 268 ( $155 \mathrm{~nm})$ and is more pronounced in $\mathrm{CD}^{+} 3^{+} \mathrm{EVs}$ and $\mathrm{CD}_{66 \mathrm{~b}^{+}} \mathrm{EVs}$. Immunological studies 269 have shown inflammatory phenotypes in granulocytes (expressing CD66b) are one of the 270 strongest discriminators between non-infected and infected individuals as well as between 
271 severity status of COVID-19 patients $^{[21]}$. The size shifts we observed correspond to the

272 moderate enrichment of smaller EVs due to cellular stress reported by others ${ }^{[22]}$. Our results

273 suggest that EV's biogenesis machinery in general especially granulocytes may have been

274 altered by SARS-CoV-2 infection causing cellular stress. Although the underlying mechanism of

275 such phenotype is yet to be determined, it is conceivable that this is partly due to the interaction

276 between SARS-CoV-2 and the endosomal sorting complexes required for EV transport ${ }^{[23]}$.

277 Moreover, size distribution correlation maps revealed a strong positive association between

278 small specialized immune cells derived EVs $\left(\mathrm{CD} 14^{+}, \mathrm{CD} 19^{+}, \mathrm{CD}^{+} 6^{+}\right.$; classical monocytes, B

279 cells, NK cells) in pre-13 days after symptoms onset, suggesting these immune compartments

280 were potentially more stressed in the early phase of COVID-19. Another highlight in our study is

281 the depletion of leukocyte-derived CD45 ${ }^{+}$EVs in early phase of mild COVID-19 (pre-13 days).

282 Although our EV data is supported by studies measuring CD45 cell counts ${ }^{[9]}$, we also observed

283 a recovery of $\mathrm{CD} 45^{+} \mathrm{EV}$ levels in the latter phase of mild COVID-19 (post-13 days). Given the

284 predominant 14 days COVID-19 isolation guidelines ${ }^{[24]}$, and the fact that these patients were all

285 mildly symptomatic our data bolsters the ability of our study to dynamically trace covid19

286 progression based on immune EVs. This also may reflect the partial recovery of host immune

287 systems as reflected by serum EVs markers. It is worth noting that by combining pre- and post-

28813 days data these small dynamic immune changes can counter-weigh each other and be lost

289 to measurements, which suggest that future work should consider infection time or disease

290 onset as a factor. Moreover, biplot cluster analysis showed insignificant variance of EVs subsets

291 distribution between age groups and sex of donors (Fig. S4).

292

293 Engineered EVs expressing SARS-CoV-2 antigens, such as ACE2 and Spike S1, have been

294 shown to perform potent functions in inhibiting coronavirus infection and serving as decoys for 295 neutralizing antibodies, respectively ${ }^{[10,11]}$. These findings not only prove the potential relevance 296 of EVs in COVID-19 progression but also highlight the possibility of SARS-CoV-2 utilizing EVs 
297 as an intermediate driver for replication and interplay with host immune defenses. Within our 298 cohort of mild COVID-19 patients, almost one-third showed significantly elevated Spike S1 ${ }^{+}$EVs 299 level in our platform when compared to healthy controls. The existence of Spike $S 1^{+} E V s$ in 300 patients' serum - which we show for the first time and which seems to peak 8-12 days post 301 symptoms - strongly support the potential of SARS-CoV-2 exploiting host EVs to act as decoys 302 for neutralizing antibodies and evade from the host immune surveillance and specific responses 303 as anticipated in an in vitro study of artificial Spike $S 1^{+} \mathrm{EVS}^{[11]}$. Moreover, the possibility to 304 detect SARS-CoV-2 Spike S1 marker from patients' serum EVs and profile their dynamics 305 during course of infection provided a novel tool for disease specific EVs phenotyping in both 306 research and clinical applications, especially in monitoring life-threatening severe COVID-19 307 progression. Throughout our panel of immune markers and endothelial marker co-stained with 308 Spike S1 across the cohort, Spike $\mathrm{S} 1^{+} \mathrm{CD} 31^{+} \mathrm{EV}$ s levels were significantly altered in pre-13 309 days COVID-19 compared to healthy controls. These data support the notion that endothelial 310 tissues (predominantly expressing CD31) were significantly influenced by SARS-CoV-2 infection, 311 but also suggesting a notable amount of Spike $S 1^{+} \mathrm{EVs}$ originate from endothelial tissues. 312 Further studies with staining of additional markers as well as future development on high 313 dimensional single EVs analysis shall provide a more conclusive representation of serum EVs 314 subsets distribution not only in COVID-19, but also other viral infections and diseases.

315 Within our dataset, a global PCA analysis of analyzed EVs subsets distribution across the 316 cohort displayed a clear stratification between healthy controls, pre-, and post- 13 days of 317 COVID-19. The partial overlap between pre-13 and post-13 days revealed the core serum EVs 318 features associated with the SARS-CoV-2 infection and might be explained by the 'long COVID' 319 observed in other follow-up studies ${ }^{[25,26]}$. Despite the overlap, post-13 days COVID-19 were less 320 separated from pre-13 days COVID-19 indicating the gradual normalization of analyzed serum 321 EVs, possibly associated with their parental cells and tissues. From the correlation clustering of 322 EVs subsets across the cohort, we could show distinct serum EVs signatures that enable 
323 differentiation between healthy controls and COVID-19 patients as well as tracing the 324 progression of COVID-19.

325 Furthermore, correlation of Spike $\mathrm{S} 1^{+} \mathrm{EVs}$ and SARS-CoV-2 specific $\lg \mathrm{A}$, IgG, IL-10, IgG1 and 326 IgG3 revealed the clinical implications of Spike $\mathrm{S}^{+} \mathrm{EVs}$ levels in the context of host immune 327 responses and disease progression. Within our dataset, Spike S1+ EVs positive patients tend to 328 show higher SARS-CoV-2 specific antibody responses and lower levels of immunosuppressive 329 IL-10, which in theory, should result in less disease deterioration. Negative correlation between 330 Spike $\mathrm{S} 1^{+} \mathrm{EV}$ s and generic viral induced $\operatorname{lgG} 1$ and $\lg \mathrm{G} 3$ responses $^{[16]}$ implies that Spike $\mathrm{S} 1^{+}$ 331 EVs levels could indicate the chances of developing SARS-CoV-2 specific responses in an early 332 predictive manner, and subsequently, enable more precise and prompt treatment decision in the 333 clinics.

335 Our functional assay of purified serum EVs in ex vivo PBMC B and T cells activation 336 demonstrated novel perspectives of the biological relevance and influences of COVID-19 337 derived serum EVs. The significant increase in $\lg A^{+} B$ cells in the presence of post-13 days 338 COVID-19 EVs indicates certain EV subsets derived from later phase of COVID-19, are partially 339 facilitating B cell antibody class switching during COVID-19 progression. Our dataset also aligns 340 with the elevated IFN- $\gamma$ levels observed in severe COVID-19 patients ${ }^{[27]}$, IFN- $\gamma$ enrichment in

$341 \mathrm{CD}^{+} \mathrm{T}$ cells in the presence of pre-13 days COVID-19 EVs but not post-13 days COVID-19 342 EVs. This dataset suggests that early in SARS-CoV-2 infection, infected host cells were 343 possibly reprogrammed to produce certain EVs subsets to promote inflammatory IFN-Y 344 production in $\mathrm{CD}^{+} \mathrm{T}$ cells. Interestingly, despite TNF elevation were observed in other clinical

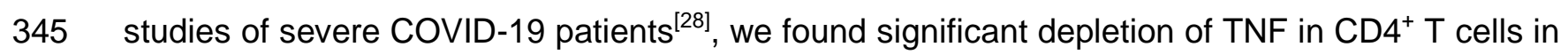
346 the presence of pre-13 days COVID-19 EVs, suggesting serum EVs from early phase of 347 COVID-19 are suppressive for TNF production in $\mathrm{CD}^{+} \mathrm{T}$ cells. Taken together, our findings 
348 provide an initial stepping-stone for the field to understand EVs signaling mediated COVID-19 349 progression.

351 With the rather small cohort size and limited markers combinations, the predictive values of 352 serum EVs could be further bolstered and fully exploited in clinical applications. Future work 353 shall consider increasing the size of cohort with the addition of severe patients from various 354 group cohorts including age, sex, genetic, geographical background, and treatment courses. 355 Moreover, to enhance the diagnostic and prognostic specificity of serum EVs in COVID-19, 356 future work shall include sampling from non-SARS-CoV-2 infected individuals such as seasonal 357 influenza patients. This approach will allow us to better stratify the EVs subsets signatures 358 between COVID-19 and general flu-like viral infections and ultimately fortify clinical predictive 359 value of EVs. Indeed, future optimization of high-dimensional spectral analyzers for EVs 360 characterization with the addition of more immune and viral markers will broaden our spectrum 361 to better trace the dynamics of host immune responses as well as disease progression. In the 362 therapeutic context, our functional assay demonstrated the feasibility of utilizing serum EVs from 363 different disease severity to manipulate host immune responses. Nevertheless, identification of 364 responsible EVs subsets and understanding their mode of action are essential for us to harvest 365 their therapeutic potentials in the future.

367 The dynamics of serum EVs subsets distribution highlighted their predictive values in the 368 perspectives of overall host immune responses and correlation between disease specific Spike $369 \mathrm{S1}^{+} \mathrm{EVS}$ and immune responses during COVID-19 progression. The study strengthens the 370 potential of serum EVs based diagnostic and prognostic, potentially therapeutic applications in 371 COVID-19, and easily transferred to other types of viral infections and cancers. 


\section{MATERIALS AND METHODS}

375 Study subjects

376 Peripheral blood was draw from healthy donors and COVID-19 patients recruited at the

377 University Hospital Zurich (Switzerland) outpatient clinic. The patients were eligible if they were 378 symptomatic at the time of inclusion, had a newly diagnosed SARS-CoV-2 infection confirmed 379 by quantitative reverse-transcriptase polymerase chain reaction (RT-qPCR), and were over 18 380 years old. Healthy donors $(n=17)$ were recruited as controls. All participants, patients and 381 healthy controls, signed a written informed consent. This non-interventional, observational study 382 was approved by the Cantonal Ethics Committee of Zurich (BASEC \#2016-01440) and 383 performed in accordance with the Declaration of Helsinki. The sample size was based on 384 availability of the samples. Investigators were blinded to disease severity, while performing 385 experiments. While the analysis was cross-sectional, the patient outcomes were recorded 386 prospectively after inclusion. Standard clinical laboratory data (CRP, LDH, complete blood count 387 with differential) was collected from the first day of hospitalization. Patients were classified 388 according to WHO guidance into mild cases $(n=20)$ in the same hospital laboratory. All healthy 389 controls were tested for SARS-CoV-2 specific IgA and IgG antibodies, and all were below the 390 diagnostic reference value. All patients received a standard clinical laboratory sampling and 391 cytokines were measured. Furthermore, samples from 20 mild COVID-19 patients and all 392 healthy subjects were processed for nano flow analysis and ex vivo activation of PBMCs. Briefly, $3933-5 \mathrm{~mL}$ of peripheral venous blood was collected from into BD Vacutainer serum clot activator $39410 \mathrm{ml}$ (367896). After collection, tubes were left vertically undisturbed on the bench for 15 395 minutes to allow blood clot and followed by centrifugation at $2,500 \mathrm{~g}$ for 10 mins at $4^{\circ} \mathrm{C}$ for 396 separation of sera. The supernatant was collected into a new tube and the serum samples were 397 stored at $4^{\circ} \mathrm{C}$ until use. Due to the limited number of available samples, certain serum samples 398 did not have enough amount for all the immunostaining panels. 
$4011 \mathrm{~mL}$ of serum samples were first diluted with $9 \mathrm{~mL}$ of PBS and concentrated using Amicon® 402 ultra-0.5 centrifugal filter devices (Millipore, Amicon® Ultra $100 \mathrm{~K}$ device) at 3,000 $\mathrm{g}$ for 30 mins 403 at $4^{\circ} \mathrm{C}$. Serum retentate (100 uL per patient) was diluted with $1.4 \mathrm{~mL}$ of PBS and subjected to 404 centrifugation at $10,000 \mathrm{~g}$ for 30 mins at $4^{\circ} \mathrm{C}$. The $10 \mathrm{k}$ pellet was resuspended in $1.5 \mathrm{~mL}$ PBS 405 and subjected ultracentrifugation at $120,000 \mathrm{~g}$ for 90 mins at $4^{\circ} \mathrm{C}$ (F50L-24x 1.5 rotor). The pellet 406 was resuspended in $50 \mathrm{uL}$ PBS and stored at $4^{\circ} \mathrm{C}$ until use.

\section{$407 \quad$ Nano flow analysis of serum EVs}

408 The configuration NanoFCM ${ }^{\circledR}$ used in the study was performed as described in another study ${ }^{[20]}$. $40910 \mathrm{uL}$ of purified serum EVs were subjected to immunofluorescence staining using antibodies 410 listed in supplementary materials (Table 1) at the concentration of 1 in $200 \mathrm{uL}$ for 1 hour at $4^{\circ} \mathrm{C}$ 411 covered in dark. Immuno-stained serum EVs were washed by ultracentrifugation at $120,000 \mathrm{~g}$ 412 for 90 mins at $4^{\circ} \mathrm{C}$, pellet was resuspended in 50 uL PBS for nano flow analysis. Monodisperse 413 silica nanoparticles of four different sizes, with modal peak sizes of $66 \mathrm{~nm}, 91 \mathrm{~nm}, 113 \mathrm{~nm}$ and $414155 \mathrm{~nm}$ were used as the size reference standard to calibrate the size distribution of EVs.

416 Ex vivo activation of PBMCs

417 Buffy coats were obtained from Blutspende Zurich, Schlieren, Switzerland (BASEC \#2019418 00837). PBMCs were isolated using Ficoll-Paque density gradient centrifugation. CD19+ B cells 419 were isolated using STEMCELL EasySep ${ }^{\mathrm{TM}}$ Release Human CD19 Positive Selection Kit 420 according to manufacturer's instruction. $\mathrm{CD}^{+} 9^{+} \mathrm{B}$ cells were activated with both human 421 recombinant IL-4 and IL-21 at $10 \mathrm{ng} / \mathrm{mL}$ (Biolegend). Remaining cell suspension were plated in 422 RPMI-1640 media supplemented in 10\% heat inactivated fetal bovine serum (FBS) (Sigma) and $4231 \%$ penicillin streptomycin/glutamine (Thermo) for 24 hours at $37^{\circ} \mathrm{C}, 5 \% \mathrm{CO}_{2}$. Cells were 424 collected at $300 \mathrm{~g}$ for 5 mins and stimulated with anti-CD3/CD28 beads at 1 bead per cell 
425 (Thermo). $50 \mathrm{uL}$ of purified serum EVs from healthy controls, pre-13 days and post-13 days mild 426 COVID-19 were added to cultured cells together with stimulatory agents.

Cell cultures transfection and flow cytometry for SARS-CoV-2 Spike S1 staining controls

429 HEK293A cells were grown in $10 \mathrm{~cm}$ tissue culture dishes in DMEM (Sigma) supplemented with

$43010 \%$ heat-inactivated FBS and 1\% penicillin/streptomycin. To generate Spike S1 expressing

431 HEK293A cells and EVs, we transfected HEK293A cells with pCMV14-3X-Flag-SARS-CoV-2 S

432 plasmid (Addgene \#145780) together with GFP plasmid (for transfection efficiency quantification)

433 and EVs were collected 24 hours post transfection from conditioned medium using serial

434 centrifugation as noted above. Transfection efficiency of HEK293A was measured by flow

435 cytometry (BD Canto II) in the FITC channel. To test the antibody binding specificity, prior to 436 immunostaining of Spike S1 on cells and EVs, molar ratios of $0: 1,1: 1,2: 1,5: 1$ of recombinant 437 Spike S1 proteins (Sino Biological, \#40591-V08H) were incubated with anti-Spike S1 for 30 438 mins at $4^{\circ} \mathrm{C}$ to neutralize unbound antibodies. Resulting staining cocktail (with range of 439 neutralizing recombinant Spike S1 proteins) were used to stain non-transfected and Spike S1 440 transfected HEK293A and EVs for 1 hour at $4^{\circ} \mathrm{C}$ covered in dark. Spike S1 signals were 441 detected by flow cytometry (HEK293A) and nano flow analyzer (EVs).

\section{Data and statistical analysis}

444 Both cells and EVs flow cytometry data were exported as FCS files and analyzed using Flowjo 445 software. Statistical analysis of clinical diagnostic and flow cytometry values were performed 446 using Graphpad (version 9.1.1, GraphPad Software, La Jolla California USA). Ordinary One447 way ANOVA, P-value $<0.05$ was considered as statistical significance. PCA and biplot were 448 generated with $\mathrm{R}$ software (version 4.0.1) using the package 'factoextra' and 'ggplot2'. 449 Spearman's correlation analyses were produced using the corrplot package and implemented 
450 hclust method. The correlations between different EVs subsets, and between different disease 451 severity group were analyzed using non-parametric Spearman correlations. The significance 452 threshold was set at alpha $<0.05$.

453

454

455 
457 We sincerely thank Prof Onur Boyman and Dr Carlo Cervia for providing critical elements for 458 this study including clinical samples, data, and insightful comments. We thank Dr Ben Peacock 459 for NanoFCM sample support. RC is supported by BBSRC (BB/N017773/2), SNF (CRSK460 3_190550; IZSEZ0_204655), and the UZH Research Priority Program (URPP Translational 461 Cancer Research). KY is supported by a Bio \& MedTech Entrepreneur Fellowship.

\section{AUTHOR CONTRIBUTIONS}

$465 \mathrm{KY}$ and RC conceived the idea and coordinated the project. RC secured funding and guided the 466 work. KY performed experiments and analyses. KY and SB performed in vitro experiments and 467 statistical data analyses. KY wrote the manuscript draft, which was then finalized by $\mathrm{KY}$ and $\mathrm{RC}$ 468 with the assistance of SB.

\section{DECLARATION OF INTERESTS}

472 All authors have declared that no conflict of interest exists.

473

474

475 
477 [1] E. Dong, H. Du, L. Gardner, Lancet Infect. Dis. 2020, 20, 533.

478 [2] Z. Wu, J. M. McGoogan, JAMA - J. Am. Med. Assoc. 2020, 323, 1239.

479 [3] V. G. Puelles, M. Lütgehetmann, M. T. Lindenmeyer, J. P. Sperhake, M. N. Wong, L. Allweiss, S. Chilla, A. Heinemann, N. Wanner, S. Liu, F. Braun, S. Lu, S. Pfefferle, A. S.

[5] K. H. W. Yim, A. Al Hrout, S. Borgoni, R. Chahwan, Cancers (Basel). 2020, $12,1$.

[6] H. BF, B.-Y. G. M, W. S, L. JC, Adv. Sci. (Weinheim, Baden-Wurttemberg, Ger. 2020, 7, DOI 10.1002/ADVS.202002596.

Schröder, C. Edler, O. Gross, M. Glatzel, D. Wichmann, T. Wiech, S. Kluge, K. Pueschel,

M. Aepfelbacher, T. B. Huber, N. Engl. J. Med. 2020, 383, 590.

[4] C. Cervia, J. Nilsson, Y. Zurbuchen, A. Valaperti, J. Schreiner, A. Wolfensberger, M. E. Raeber, S. Adamo, S. Weigang, M. Emmenegger, S. Hasler, P. P. Bosshard, E. De Cecco, E. Bächli, A. Rudiger, M. Stüssi-Helbling, L. C. Huber, A. S. Zinkernagel, D. J. Schaer, A. Aguzzi, G. Kochs, U. Held, E. Probst-Müller, S. K. Rampini, O. Boyman, J. Allergy Clin. Immunol. 2021, 147, 545.

[8] S. AM, L.-I. E, G. A, D. A, D. G, P. B, O. X, S. N, F. KP, S. O, D. N, J. Extracell. vesicles 2021, 10, DOI 10.1002/JEV2.12130.

[9] M. Jin, N. Shi, M. Wang, C. Shi, S. Lu, Q. Chang, S. Sha, Y. Lin, Y. Chen, H. Zhou, K.

[10] F. Cocozza, N. Névo, E. Piovesana, X. Lahaye, J. Buchrieser, O. Schwartz, N. Manel, M. Tkach, C. Théry, L. Martin-Jaular, J. Extracell. Vesicles 2020, 10, DOI 10.1002/jev2.12050.

[11] Z. Troyer, N. Alhusaini, C. O. Tabler, T. Sweet, K. I. L. Carvalho, D. M. Schlatzer, L. Carias, C. L. King, K. Matreyek, J. C. Tilton, J. Extracell. Vesicles 2021, 10, e12112.

[12] J. H. Levy, T. Iba, E. E. Gardiner, Arterioscler. Thromb. Vasc. Biol. 2021, 41, 1774.

[13] A. Birnhuber, E. Fliesser, G. Gorkiewicz, M. Zacharias, B. Seeliger, S. David, T. Welte, J. Schmidt, H. Olschewski, M. Wygrecka, G. Kwapiszewska, Eur. Respir. J. 2021, 2100377.

509 [16] G. Vidarsson, G. Dekkers, T. Rispens, Front. Immunol. 2014, 5, DOI 
10.3389/fimmu.2014.00520.

[17] Y. Fujita, T. Hoshina, J. Matsuzaki, Y. Yoshioka, T. Kadota, Y. Hosaka, S. Fujimoto, H. Kawamoto, N. Watanabe, K. Sawaki, Y. Sakamoto, M. Miyajima, K. Lee, K. Nakaharai, T. Horino, R. Nakagawa, J. Araya, M. Miyato, M. Yoshida, K. Kuwano, T. Ochiya, J. Extracell. Vesicles 2021, 10, e12092.

[18] K. Mao, Q. Tan, Y. Ma, S. Wang, H. Zhong, Y. Liao, Q. Huang, W. Xiao, H. Xia, X. Tan, P. Luo, J. Xu, D. Long, Y. Jin, Cell Death Dis. 2021, 12, 541.

[19] C. Balbi, J. Burrello, S. Bolis, E. Lazzarini, V. Biemmi, E. Pianezzi, A. Burrello, E. Caporali, L. G. Grazioli, G. Martinetti, T. Fusi-Schmidhauser, G. Vassalli, G. Melli, L. Barile, EBioMedicine 2021, 67, 103369.

[20] Y. Tian, M. Gong, Y. Hu, H. Liu, W. Zhang, M. Zhang, X. Hu, D. Aubert, S. Zhu, L. Wu, X. Yan, J. Extracell. Vesicles 2020, DOI 10.1080/20013078.2019.1697028.

[21] J. Vitte, A. B. Diallo, A. Boumaza, A. Lopez, M. Michel, J. Allardet-Servent, S. Mezouar, Y. Sereme, J. M. Busnel, T. Miloud, F. Malergue, P. E. Morange, P. Halfon, D. Olive, M.

[22] O. G. de Jong, M. C. Verhaar, Y. Chen, P. Vader, H. Gremmels, G. Posthuma, R. M. Leone, J. L. Mege, J. Infect. Dis. 2020, 222, 1985.

Schiffelers, M. Gucek, B. W. M. van Balkom, J. Extracell. Vesicles 2012, 1, DOI 10.3402/jev.v1i0.18396.

[23] M. V. Veettil, B. Kumar, M. A. Ansari, D. Dutta, J. lqbal, O. Gjyshi, V. Bottero, B. Chandran, J. Virol. 2016, 90, 3860.

[24] K. A. Walsh, S. Spillane, L. Comber, K. Cardwell, P. Harrington, J. Connell, C. Teljeur, N. Broderick, C. F. de Gascun, S. M. Smith, M. Ryan, M. O’Neill, J. Infect. 2020, 81, 847.

[25] M. Merad, J. C. Martin, Nat. Rev. Immunol. 2020, 20, 355.

[26] K. E. Bernstein, Z. Khan, J. F. Giani, D. Y. Cao, E. A. Bernstein, X. Z. Shen, Nat. Rev. Nephrol. 2018, 14, 325.

[27] I. E. Galani, N. Rovina, V. Lampropoulou, V. Triantafyllia, M. Manioudaki, E. Pavlos, E. Koukaki, P. C. Fragkou, V. Panou, V. Rapti, O. Koltsida, A. Mentis, N. Koulouris, S. Tsiodras, A. Koutsoukou, E. Andreakos, Nat. Immunol. 2021, 22, 32. 
543 Table 1. Demographic and clinical characteristics of the patient cohort.

545 Figure 1. Characterization of immune serum EVs in healthy controls and mild COVID-19 546 patients. (A) Schematic outline of EVs profiling from denoted human samples. (B) Size 547 distribution quantification of serum EVs from denoted human samples and different EV subsets, 548 with size reference beads with a mixture of four modal sizes of $66 \mathrm{~nm}$ (small), $91 \mathrm{~nm}$ (medium), $549113 \mathrm{~nm}$ (large), $155 \mathrm{~nm}$ (extra-large). Representative side scatter histogram of size reference 550 beads in (B) and total serum EVs from denoted human samples on the right. (C, D) 551 Quantification of total serum EVs and CD45+ EVs in denoted human samples at days of 552 reported symptom onset. (E) Spearman's rank correlation matrix of size distribution of serum 553 EVs subsets between healthy donors and mild COVID-19 patients. One-way ANOVA, p $<0.05$ *, $554 p<0.01^{* *}, p<0.005^{* * *}$.

Figure 2. Characterization of Sars-Cov-2 Spike $S 1+$ serum EVs in healthy controls and mild COVID-19 patients. (A) Quantification of Spike S1+, Spike S1+CD31+ and CD31+ serum EVs in denoted human samples, dark blue (Spike $S 1^{+}$EVs positive). (B) Size distribution quantification of serum EVs from denoted human samples and indicated EV subsets. (C) Bi-plot and principal component analysis of set of serum EVs markers defining healthy and mild COVID-19 status. (D) Spearman's rank correlation matrix of serum EVs subsets between healthy donors and mild COVID-19 patients. One-way ANOVA, $p<0.05^{*}, p<0.01^{* *}, p<0.005^{* * *}$.

Figure 3. Correlation of Sars-Cov-2 Spike S1+ serum EVs with host immune responses in healthy controls and mild COVID-19 patients. (A) Quantification of Sars-Cov-2 Spike S1+ and Sars-Cov-2 Spike S1+CD31+EVs in denoted human samples at days after reported symptom onset. (B, C) Quantification of soluble Sars-Cov-2 specific immunoglobulins in denoted human samples at days after reported symptom onset. (D) Quantification of soluble interleukin-10 levels in denoted human samples at days after reported symptom onset. (E) Spearman's correlation of level of Spike S1+ serum EVs to Spike S1+CD31+EVs, soluble IgG1, IgG3 and 571 interleukin-10 levels.

573 Figure 4. Mild COVID-19 patients derived serum EVs affect healthy PBMCs responses ex vivo 574 (A) Schematic outline of ex vivo healthy PBMCs activation in the presence of PBS control and 575 serum EVs from healthy donors and mild COVID-19 patients. (B-D) Quantification of different 
576 subsets expansion, activation, cytokine production ( $T$ cells) (C, D) and class switch 577 recombination (B cells) (B). One-way ANOVA, $p<0.05^{*}, p<0.01^{* *}, p<0.005^{* * *}$.

SUPPLEMENTARY FIGURE LEGENDS

Figure S1. Nanoflow control and experimental setup. (A) Representative nanoflow gating 582 strategies of EVs, HEK293A derived EVs (control) and representative patient derived serum 583 EVs. (B) Representative back gating strategies to determine size distribution of EV subsets 584 using size reference beads with mixture of four modal sizes, $66 \mathrm{~nm}$ (small), $91 \mathrm{~nm}$ (medium), $585113 \mathrm{~nm}$ (large), $155 \mathrm{~nm}$ (extra-large). (C) Quantification of CD45 ${ }^{+}$serum EVs in healthy controls 586 and mild COVID-19 patients. (D) Quantification of size distribution of $\mathrm{CD}^{2} 8^{+}, \lg \mathrm{A}^{+}, \lg \mathrm{G}^{+}$serum 587 EVs in healthy controls and mild COVID-19 patients.

Figure S2. Binding specificity of Sars-CoV-2 Spike S1 antibodies. (A) Representative flow gating strategies of HEK293A co-transfected with GFP and Spike S1 plasmid after 24 hours. (B) Competition of anti-Spike S1 binding in HEK293A in (A) with addition of recombinant Spike S1 proteins in denoted molar ratio. (C) Representative flow gating strategies of EVs derived from HEK293A co-transfected with GFP and Spike S1 plasmid after 24 hours and competition of antiSpike S1 binding in EVs with addition of recombinant Spike S1 proteins in denoted molar ratio.

Figure S3. (A) Representative flow gating strategies of patient derived serum EVs for Spike S1 and CD31. (B) Quantification of Spike $S 1^{+} \mathrm{CD} 45^{+}$, Spike $S 1^{+} \mathrm{CD} 38^{+}$, Spike $\mathrm{S} 1^{+} \mathrm{CD} 56^{+}$, Spike $598 \mathrm{~S}^{+} \lg \mathrm{A}^{+}$, Spike $\mathrm{S} 1^{+} \operatorname{lgG}{ }^{+}$, Spike $\mathrm{S} 1^{+} \mathrm{CD} 66 \mathrm{~b}^{+}$serum EVs in in healthy controls and mild COVID-19 599 patients.

Figure S4. PCA plot clustering of serum EVs samples based on age (A) and sex (B). 
Table 1. Demographic and clinical characteristics of the patient cohort.

\begin{tabular}{|c|c|c|c|}
\hline & Healthy & Mild COVID-19 & \\
\hline No. of samples & 17 & 20 & \\
\hline Median age [years] & 31 & 34 & \\
\hline Gender $(\mathrm{M} / \mathrm{F})$ & $7 / 10$ & $11 / 9$ & \\
\hline Time since symptom onset (days) & - & $11 \pm 5.01996$ & \\
\hline Laboratories values & & & $P$ value \\
\hline Hemoglobin(mean $\pm \mathrm{SD},[\mathrm{g} / \mathrm{l}])$ & $143.13 \pm 11.76$ & $81.35 \pm 75.79$ & 0.0028 \\
\hline Absolute platelet count(mean $\pm \mathrm{SD},[\mathrm{G} / \mathrm{l}])$ & $252 \pm 50.71$ & $243.25 \pm 61.79$ & ns \\
\hline Total white blood cell count mean $\pm \mathrm{SD},[\mathrm{G} / \mathrm{l}])$ & $6.06 \pm 1.73$ & $5.13 \pm 1.14$ & ns \\
\hline Monocytes $($ mean $\pm \mathrm{SD},[\mathrm{G} / \mathrm{l}])$ & $0.45 \pm 0.16$ & $0.46 \pm 0.1$ & ns \\
\hline Neutrophils(mean \pm SD, [G/l]) & $3.61 \pm 1.29$ & $2.64 \pm 0.91$ & 0.0129 \\
\hline Eosinophils(mean $\pm \mathrm{SD},[\mathrm{G} / \mathrm{l}]$ ) & $0.1 \pm 0.05$ & $0.12 \pm 0.09$ & ns \\
\hline Basophils(mean \pm SD, [G/l]) & $0.05 \pm 0.01$ & $0.03 \pm 0.01$ & 0.0015 \\
\hline Lymphocytes(mean \pm SD, [G/l]) & $1.84 \pm 0.56$ & $1.85 \pm 0.56$ & ns \\
\hline $\begin{array}{l}\text { CD3- CD56bright CD16dim NK cells } \\
\text { (mean } \pm \text { SD, [cells/ul]) }\end{array}$ & $18.47 \pm 5.35$ & $12.1 \pm 5.7$ & ns \\
\hline $\begin{array}{l}\text { CD3- CD56dim CD16bright NK cells } \\
\text { (mean } \pm \text { SD, [cells/ul]) }\end{array}$ & $164.59 \pm 119.87$ & $248.25 \pm 131.75$ & ns \\
\hline CD4+ T cells(mean $\pm \mathrm{SD},[$ cells/ul]) & $376.24 \pm 468.03$ & $780.3 \pm 290.92$ & 0.0029 \\
\hline CD19+ B cells(mean \pm SD, [cells/ul]) & $101.41 \pm 130.44$ & $189.4 \pm 103.36$ & 0.0282 \\
\hline C-reactive protein (mean $\pm \mathrm{SD},[\mathrm{mg} / \mathrm{l}])$ & $1.04 \pm 0.97$ & $1.74 \pm 1.89$ & ns \\
\hline $\mathrm{LDH}($ mean $\pm \mathrm{SD},[\mathrm{U} / \mathrm{l}])$ & $332.24 \pm 53.88$ & $342.39 \pm 79.04$ & ns \\
\hline IL-6(mean \pm SD, $[\mathrm{pg} / \mathrm{ml}])$ & $0.47 \pm 0.57$ & $2.51 \pm 4.24$ & ns \\
\hline IL-10 (mean \pm SD, [pg/ml]) & $1.15 \pm 1.3$ & $1.64 \pm 2.19$ & ns \\
\hline IFNy (mean \pm SD, [pg/ml]) & $0.88 \pm 1.68$ & $1.89 \pm 2.64$ & ns \\
\hline $\mathrm{TNF} \alpha($ mean $\pm \mathrm{SD},[\mathrm{pg} / \mathrm{ml}])$ & $7.32 \pm 3.04$ & $8.36 \pm 3.49$ & ns \\
\hline Anti-CoV-2 lgA(mean $\pm S D,[\mu g / m L])$ & $0.34 \pm 0.18$ & $4.85 \pm 7.2$ & 0.0144 \\
\hline Anti-CoV-2 lgG(mean $\pm S D,[\mu \mathrm{g} / \mathrm{mL}])$ & $0.27 \pm 0.15$ & $1.03 \pm 0.92$ & 0.0019 \\
\hline \multicolumn{4}{|l|}{ Comorbidities } \\
\hline Hypertonia - no. (\%) & - & - & \\
\hline Diabetes - no. (\%) & - & - & \\
\hline Heart disease - no. (\%) & - & $1(5 \%)$ & \\
\hline Lung disease - no. (\%) & - & - & \\
\hline Malignancy - no. (\%) & - & - & \\
\hline Immunosuppression - no. (\%) & - & - & \\
\hline Kidney disease - no. (\%) & - & - & \\
\hline Cerebrovascular disease - no. (\%) & - & - & \\
\hline M Crohn - no. (\%) & - & $1(5 \%)$ & \\
\hline Allergic asthma - no. (\%) & - & $2(10 \%)$ & \\
\hline Hypothyreose - no. (\%) & $1(6 \%)$ & $3(15 \%)$ & \\
\hline
\end{tabular}



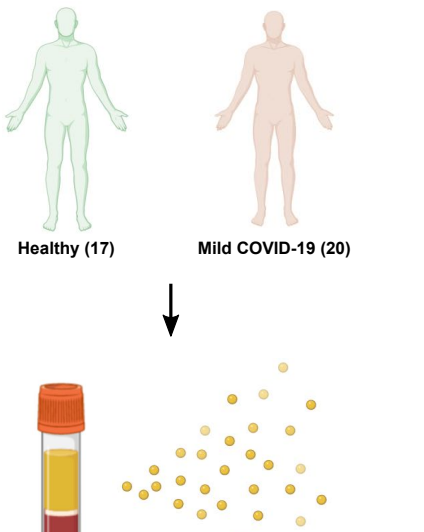

Serum Immunostaining of total serum EVs sil $\rightarrow$ ill

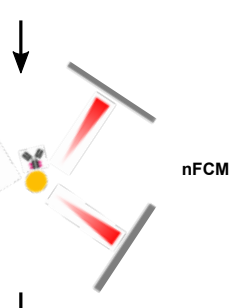

\section{IIII}

Size \& Concentration Surface markers Clustering

C

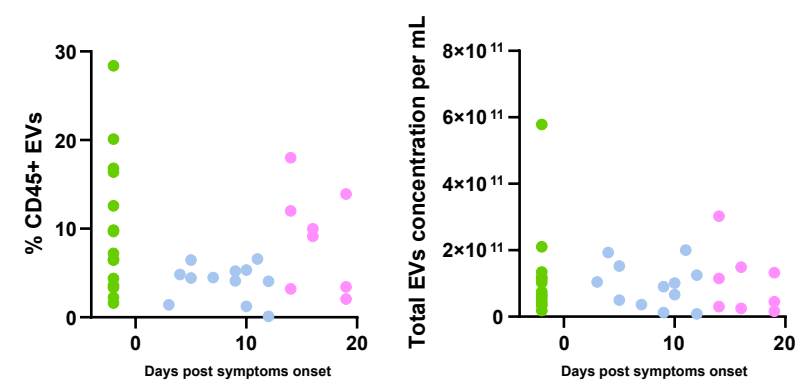

E
Pan EVs and pan leukocytes derived EVs
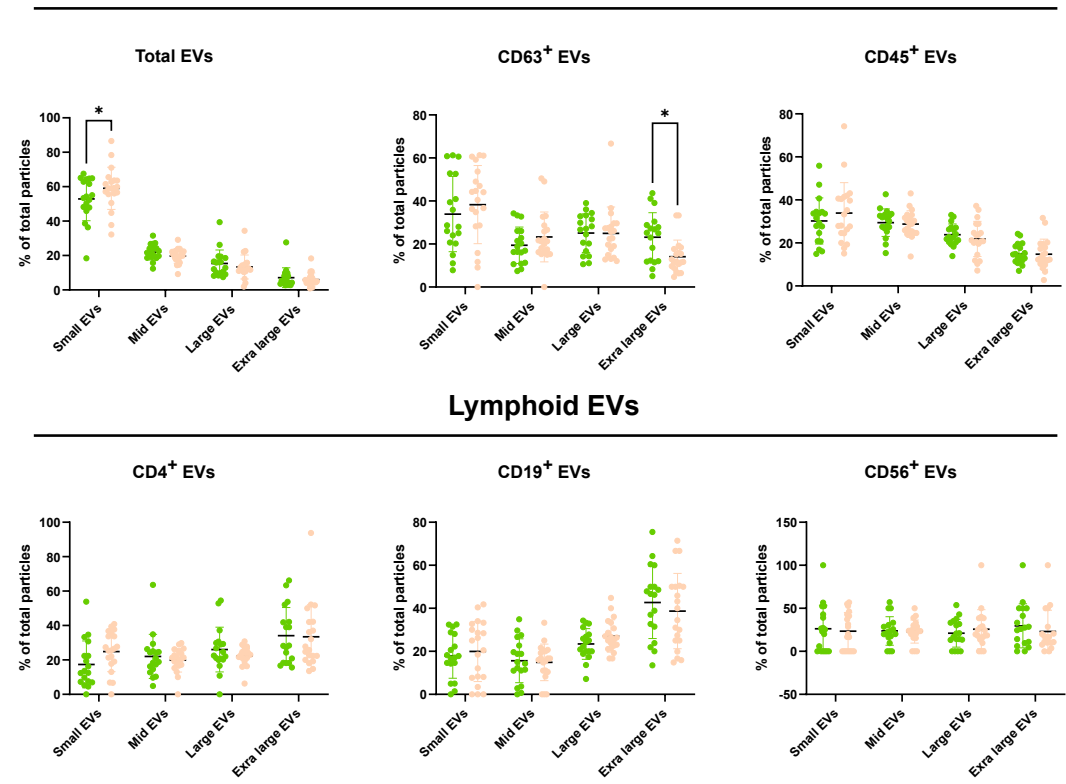

Myeloid EVs
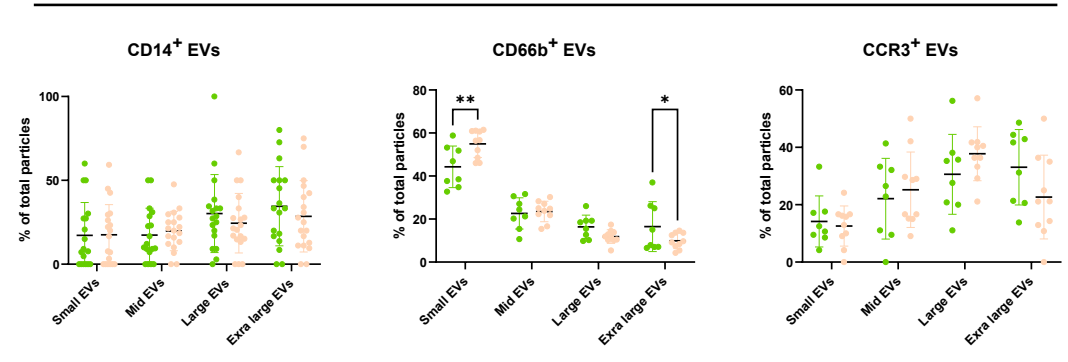

D
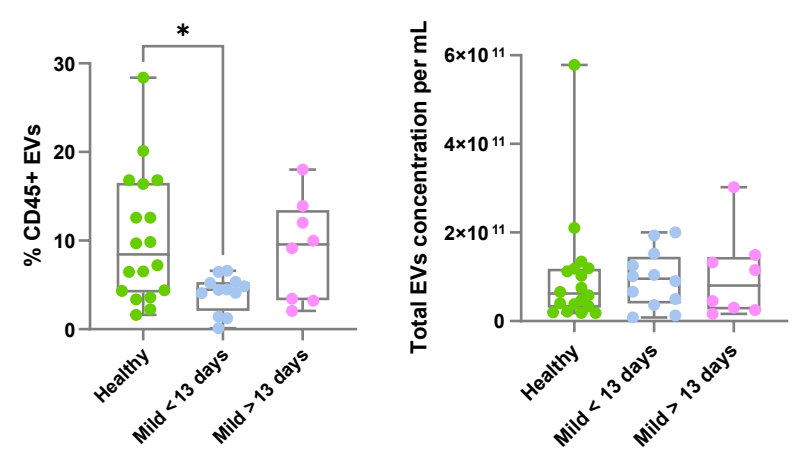

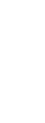

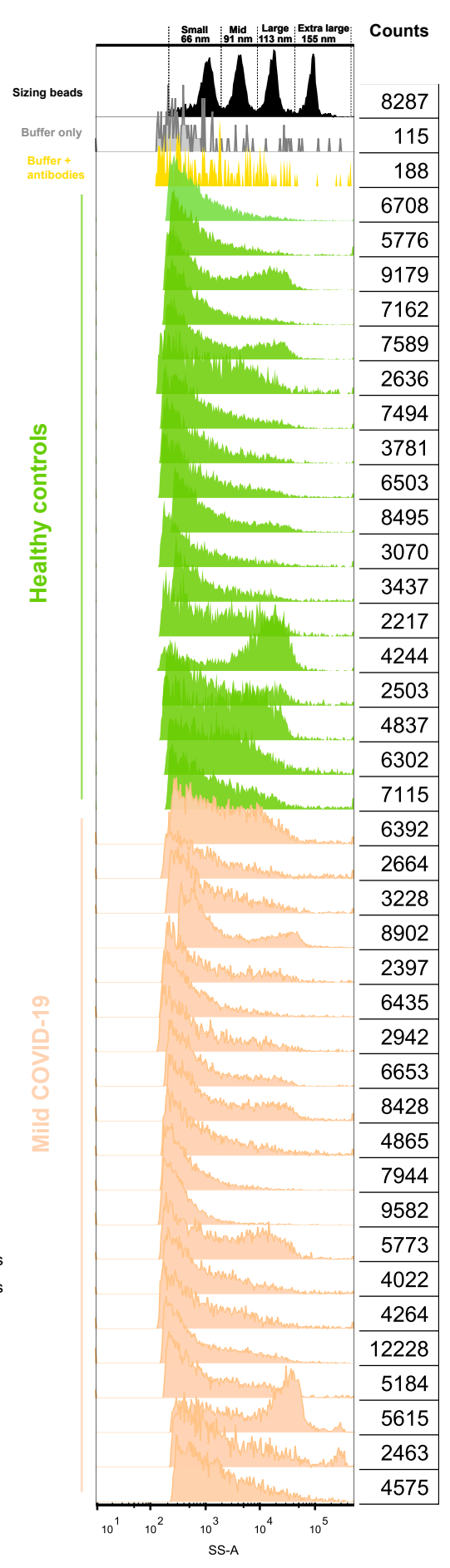

Healthy

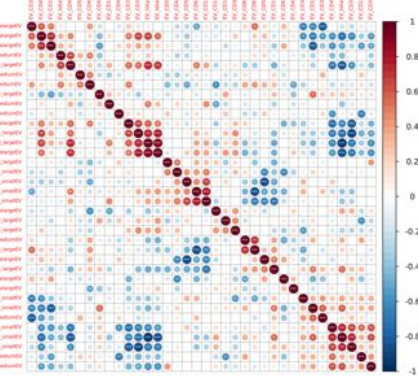

EVs subset size correlation

Mild $<13$ days

Mild $>13$ days

Healthy

Mild $<13$ days

Mild $>13$ days 
A
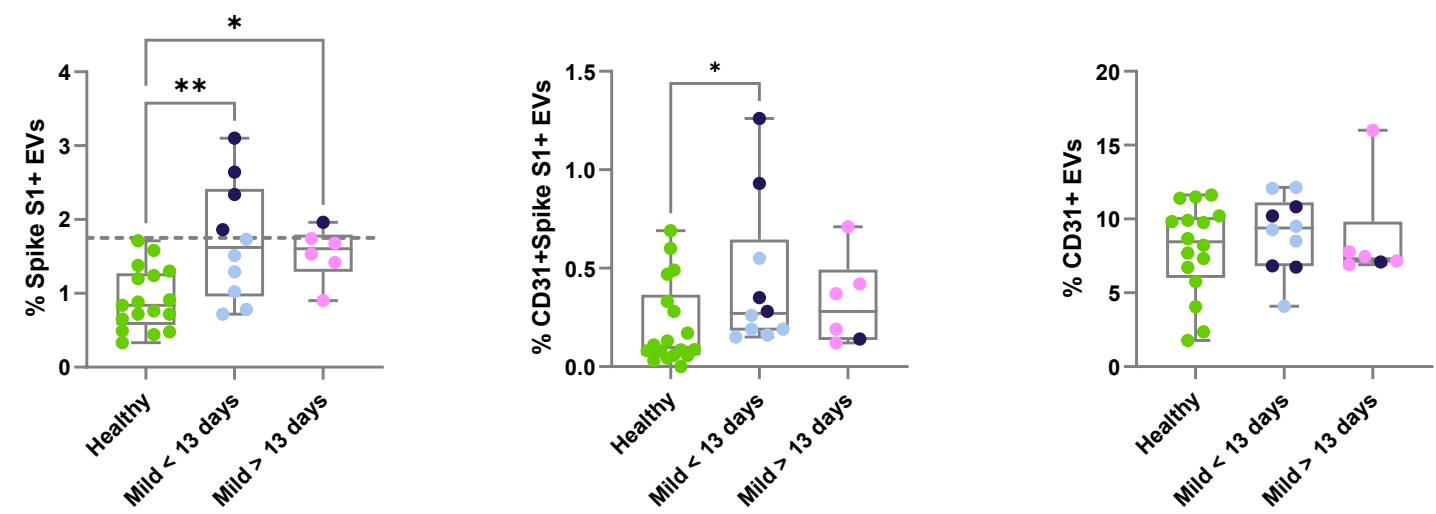

- Healthy

Mild $<13$ days

- Mild $>13$ days

- EV Spike S1 ${ }^{\text {high }}$

B

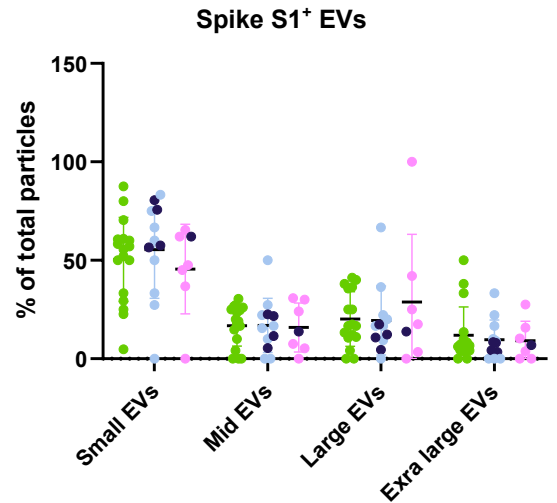

C

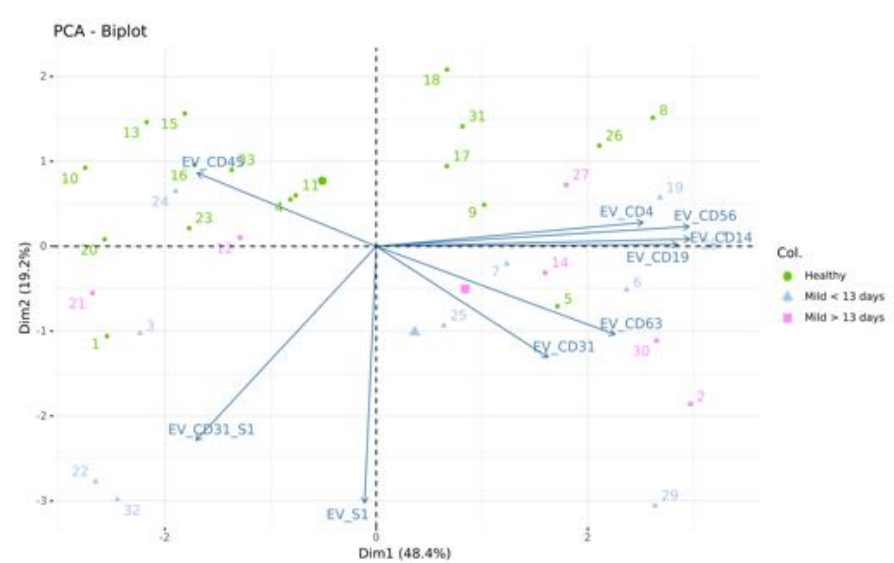

Spike $\mathrm{S} 1^{+} \mathrm{CD} 31^{+} \mathrm{EVs}$

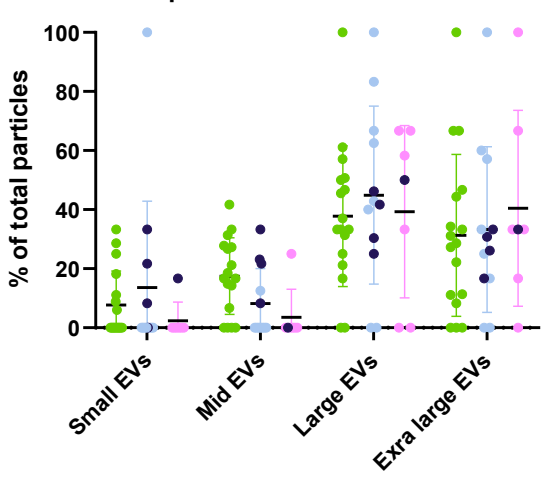

D

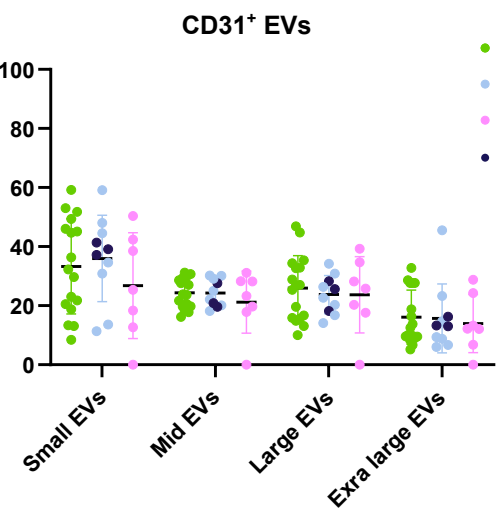

- Healthy

Mild $<13$ days

Mild $>13$ days

E

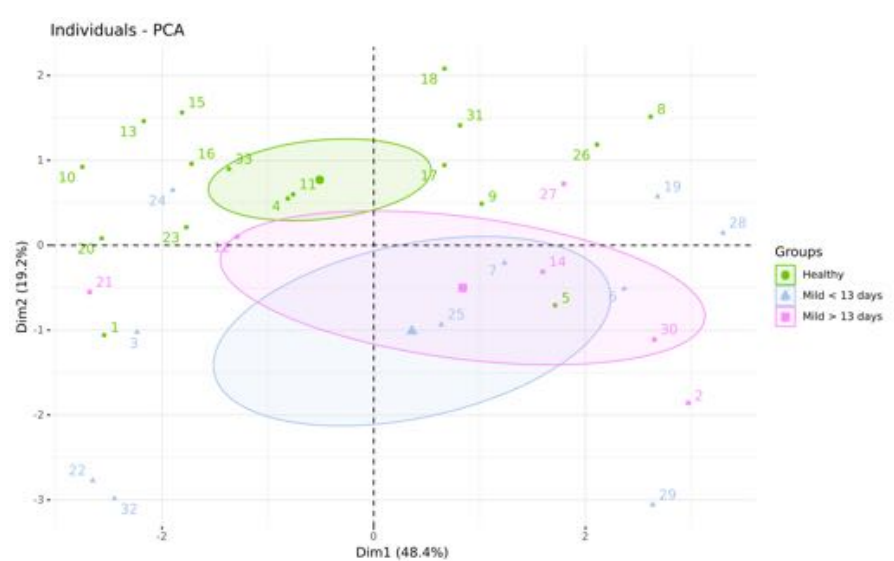
EVs subsets correlation

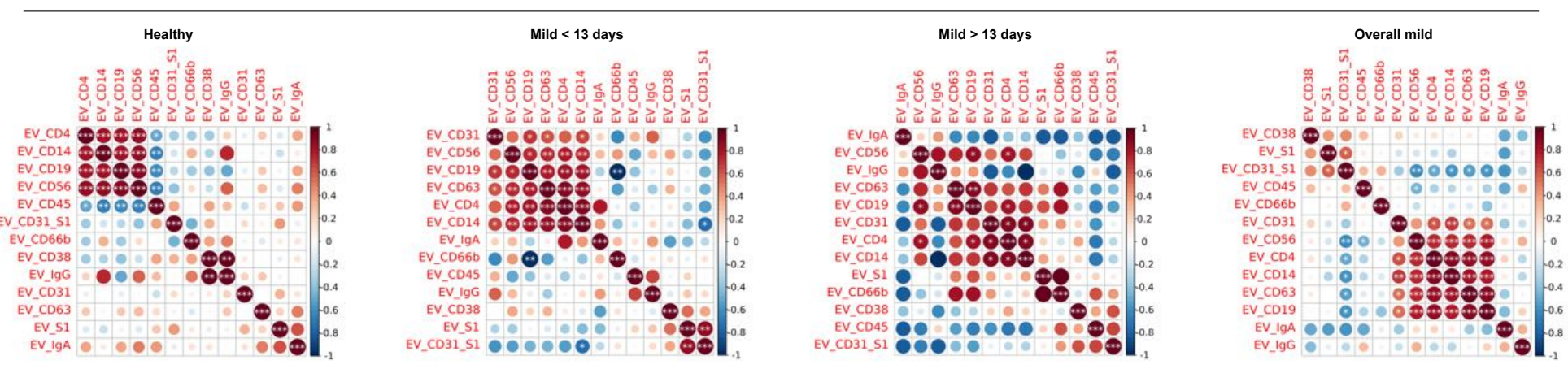


A
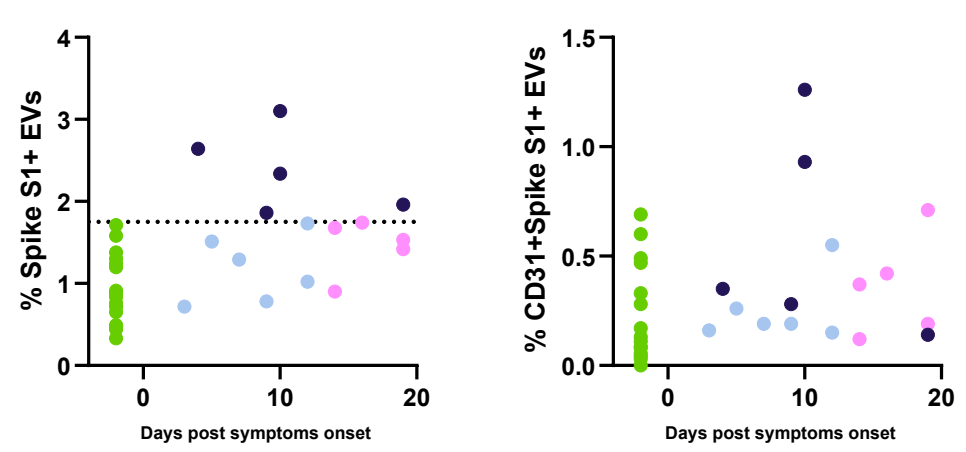

B

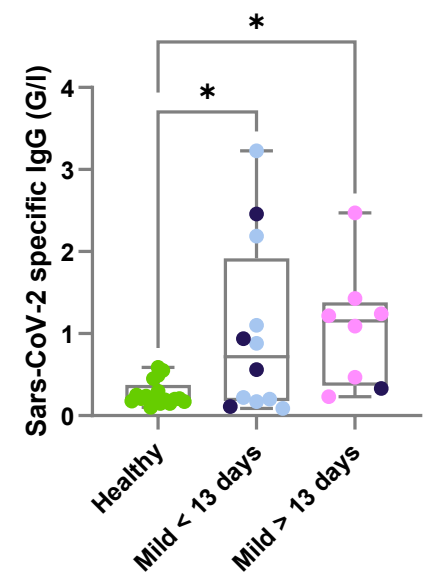

C

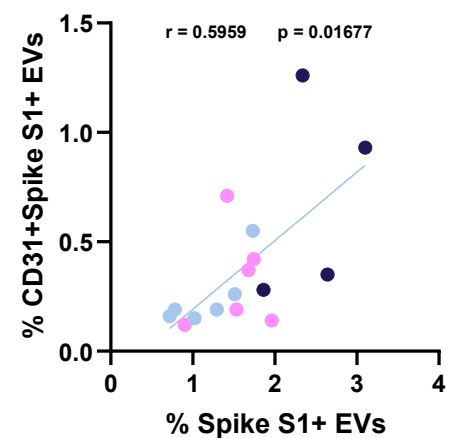

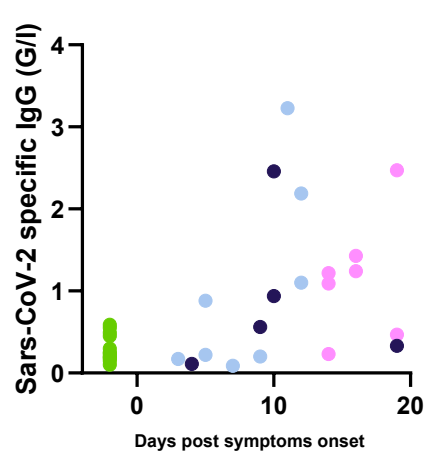
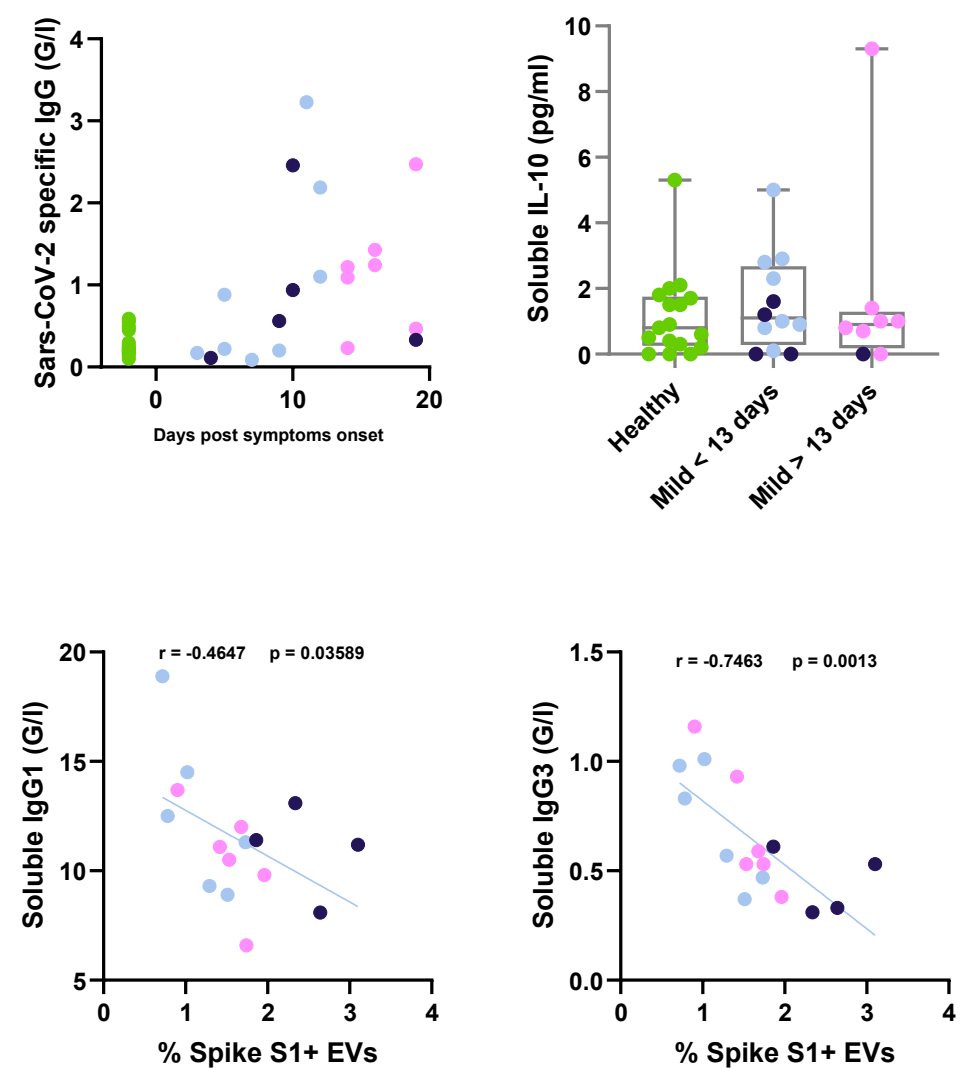

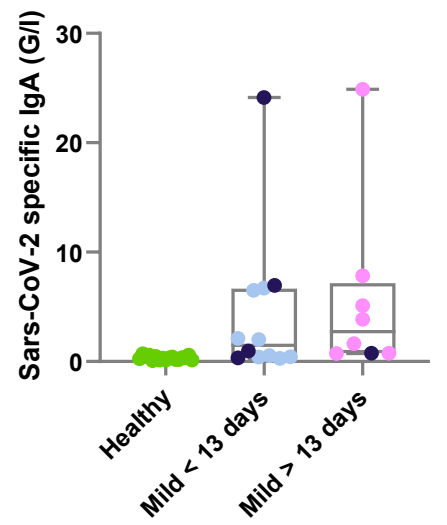

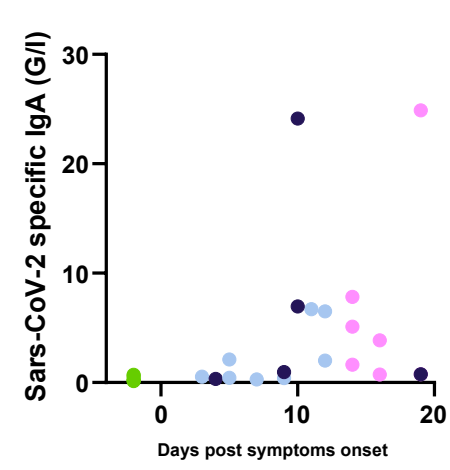

- Healthy
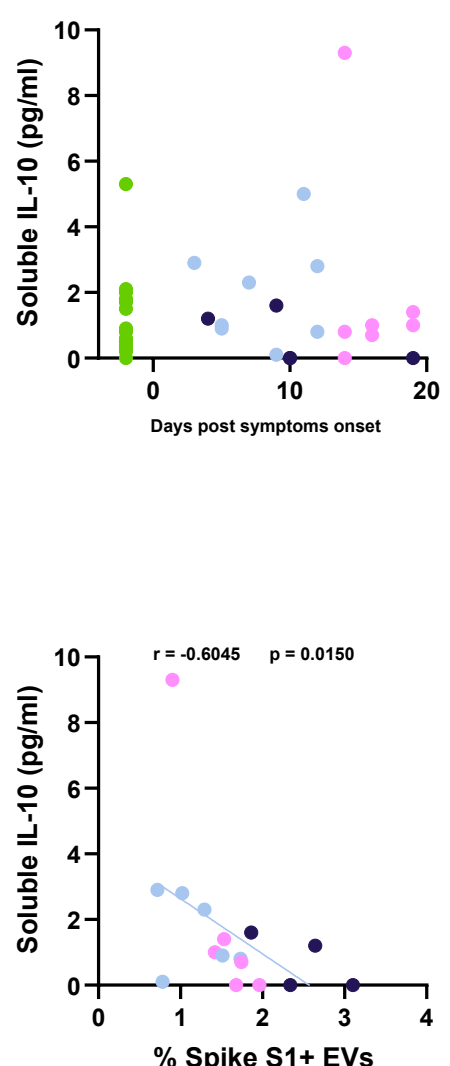

Healthy
Mild $<13$ days

Mild $>13$ days

- EV Spike S1 $1^{\text {high }}$

Mild $<13$ days

Mild $>13$ days

- EV Spike S1 ${ }^{\text {high }}$

Mild $<13$ days

Mild $>13$ days

- EV Spike $S 1^{\text {high }}$ 


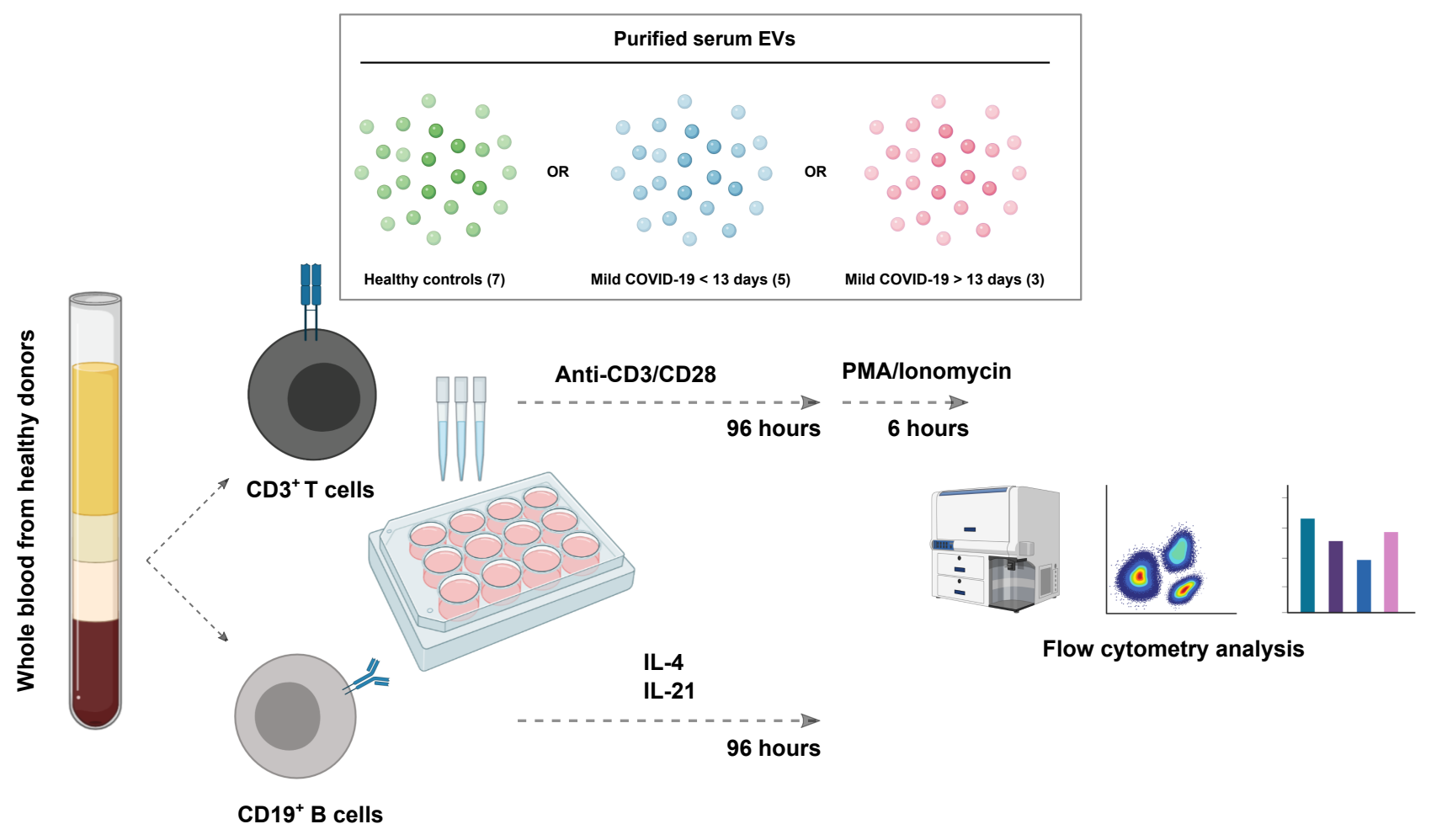

B
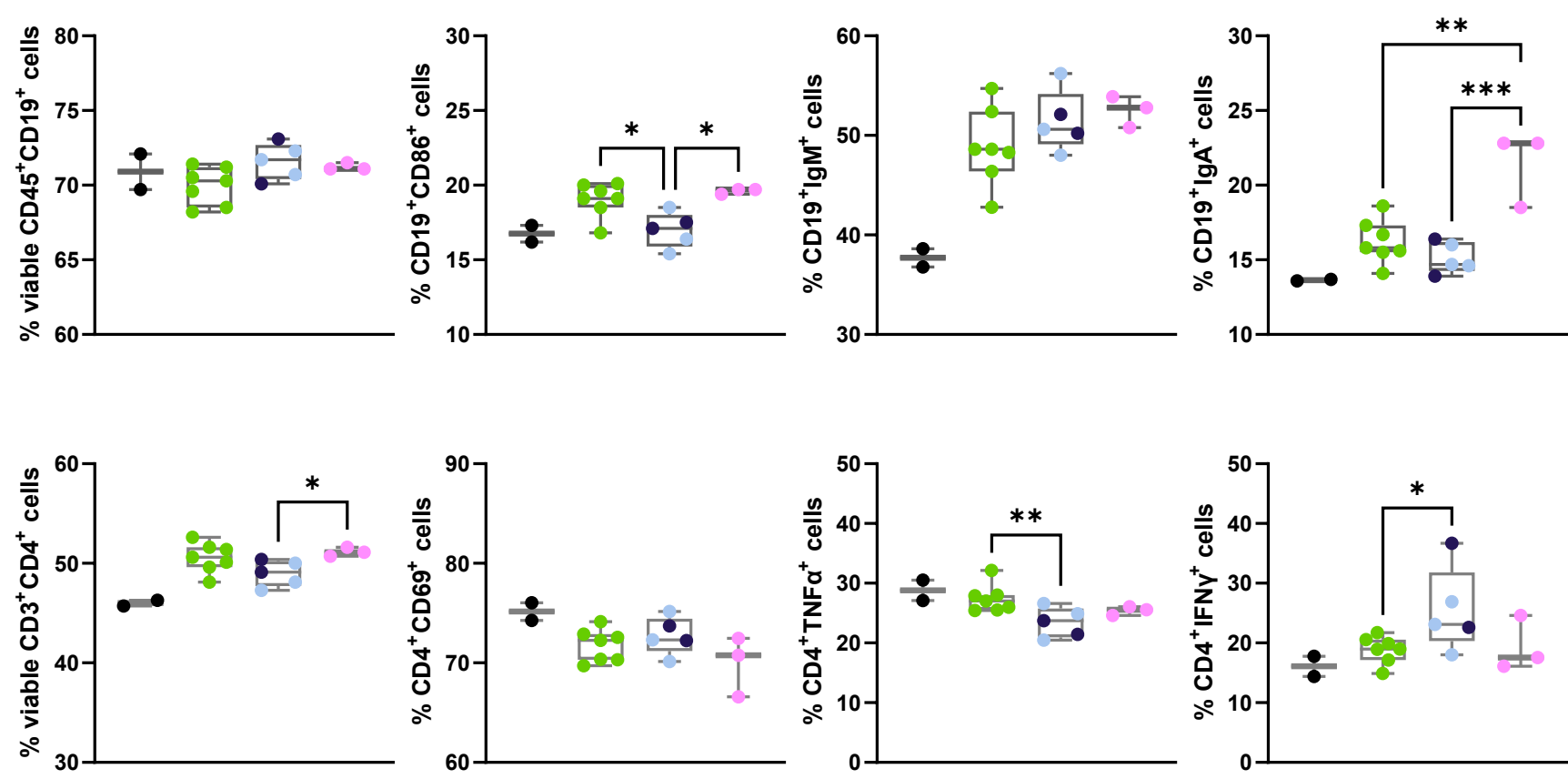

D

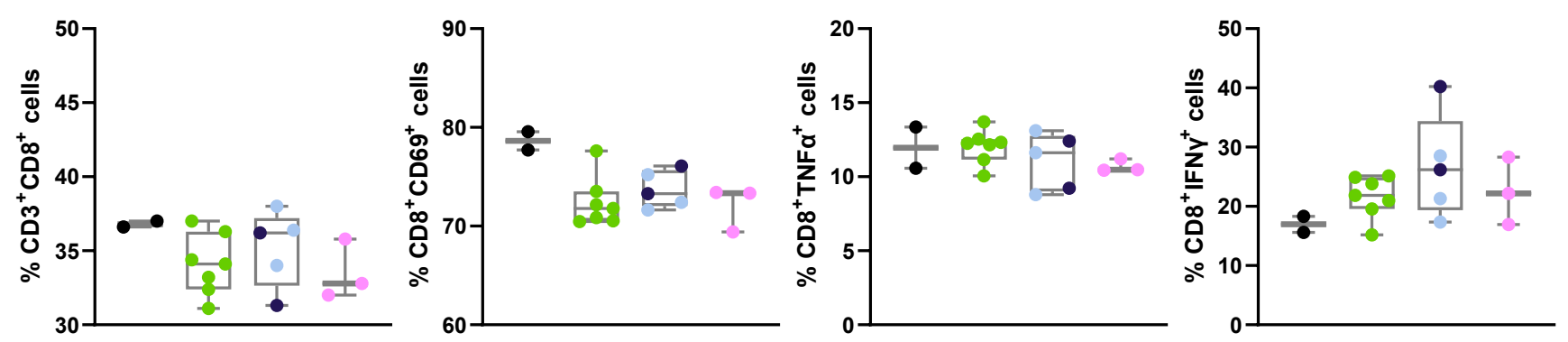

